\title{
REDUCED ORDER MODELS FOR CLOSED LOOP CONTROL: COMPARISON BETWEEN POD, BPOD, AND GLOBAL MODES
}

\author{
A. Barbagallo, ${ }^{1,2}$ D. Sipp ${ }^{2}$, and P. J. Schmid ${ }^{1}$ \\ ${ }^{1}$ Laboratoire d'Hydrodynamique (LadHyX) \\ CNRS-Ecole Polytechnique \\ Palaiseau, France \\ ${ }^{2}$ ONERA-DAFE
}

Rue des Vertugardins 8, Meudon, France

\begin{abstract}
Linear Quadratic Gaussian (LQG) control is a promising tool to control flows. Initially developed for systems of moderate size, it is not directly applicable to fluid mechanics problems, requiring a reduced model. Three popular ways of reducing the system will be presented and their ability to control the global instability of an incompressible cavity flow will be studied. A comparison between reduced models based on global modes, Proper Orthogonal Decomposition (POD) modes and Balanced POD (BPOD) modes permits to discuss the relevant quantities to be captured by the reduced model to insure a successful control.
\end{abstract}

\section{INTRODUCTION}

Over the last decade, substantial effort has been placed on suppressing instabilities in unstable flows. To achieve this goal, the tools developed in control theory, such as optimal control, seem quite promising. However, since the typical size of discretized problems in fluid mechanics is generally too high to apply these techniques directly, a reduced-order model (ROM) of the flow is needed.

A reduced model may be obtained by a Petrov-Galerkin projection of the discretized equations onto either global [1], POD [2], or balanced modes [3]. Each of these bases captures a different feature of the flow and thus accomplishes a different goal. For control problems, the relevant quantity of interest is the inputoutput relation which can be optimally captured by balanced truncation [4]. This method, which determines modes that show the same amount of controllability and observability, became recently computationally affordable with the so-called BPOD technique [5].

This is an Open Access article distributed under the terms of the Creative Commons Attribution-Noncommercial License 3.0, which permits unrestricted use, distribution, and reproduction in any noncommercial medium, provided the original work is properly cited. 
In this paper, reduced models based on global modes, POD, and BPOD modes will be determined. Since the input-output relation is a key parameter for control problems, the ability of each model to capture this quantity will be evaluated. Then, the capacity of stabilizing the flow with closed-loop control will be demonstrated. In conclusion, a comparison concerning the minimal number of modes required by each reduced model to stabilize the flow is made.

\section{CONFIGURATION AND MATHEMATICAL MODELING}

Let consider the flow over an open square cavity (Fig. 1). The flow is incompressible and the Reynolds number based on the cavity depth and the inflow velocity is 7500. This flow is subject to a low-frequency unsteadiness which can be modeled by a global instability approach [6]. According to the global stability theory, the linear growth of perturbations is studied. The base flow, solution of the steady Navier-Stokes equations, is computed using a Newton method and shown in Fig. 2. For this Reynolds number, the base flow is globally unstable. A feedback control strategy is implemented to suppress the instabilities, using a normal blowing and suction actuator located at the leading edge of the cavity and a shear-stress sensor at the trailing edge.

The linearized Navier-Stokes equations are discretized with finite elements using P2 (respectively, P1) elements for the velocity components $\mathbf{u}=(u, v)^{\mathrm{T}}$ (respectively, the pressure component $p$ ). This results in approximately $n$ $\approx 900000$ degrees of freedom. The discretized problem can be written as:

$$
\begin{aligned}
\mathrm{Q} \frac{d \mathrm{X}}{d t} & =\mathrm{AX}+\mathrm{C} c ; \\
m & =\mathrm{MX}
\end{aligned}
$$

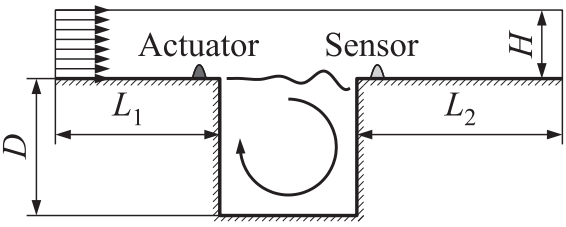

Figure 1 Sketch of the cavityl

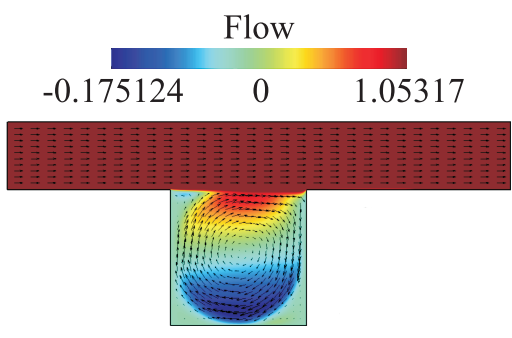

Figure 2 Base flow. (Refer Barbagallo et al., p. 504.) 
where $\mathbf{X}=(\mathbf{u}, p)^{\mathrm{T}} ; \mathbf{A}$ is the linearized Navier-Stokes operator; $\mathbf{C}$ is the control matrix; $M$ is the measurement matrix; and $Q$ stands for the weight matrix arising from the finite elements discretization. The use of a Single Input Single Output (SISO) framework implies that C (respectively, M) is of dimension $(n \times 1)$ (respectively, $(1 \times n))$; $c$ is the scalar control law; and $m$ is the scalar measure both depending on time. Note that $\mathrm{X}^{\mathrm{T}} \mathrm{QY}$ represent the energy based scalar product between $\mathrm{X}$ and $\mathrm{Y}$.

\section{MODEL REDUCTION}

In order to apply LQG control, it is necessary to reduce the size of the system. This is achieved by a Petrov-Galerkin projection of Eq. (1) onto a particular basis. In general case, this basis is bi-orthogonal: the basis $\mathrm{V}$ (so-called "direct basis") verifies the relation $\mathrm{W}^{*} \mathrm{QV}=\mathrm{I}$ where $\mathrm{W}$ is the adjoint basis.

To control global instabilities, it is required to model the unstable subspace and may also be necessary to model the stable subspace. In this study, these subspaces will be modeled separately. The unstable global modes will be considered for the unstable subspace. For the stable subspace, models based on global, POD, and BPOD modes will be computed and compared.

\subsection{Global Modes}

Let consider global modes of the following form:

$$
\mathbf{X}(x, y, t)=\tilde{\mathbf{X}}(x, y) e^{\lambda t}, \quad \lambda=\sigma+i \omega
$$

which are solutions of the eigenvalue problem $\lambda \mathrm{QX}=\mathrm{A} \tilde{\mathrm{X}}$. This equation is solved with a shift-invert Arnoldi algorithm. The least-stable part of the global spectrum is displayed in Fig. 3 .

Four unstable modes (with positive growth rate $\sigma>0$ ) are shown by empty signs. These modes, cast into the $(n \times(2 \times 4))$ matrix $\mathrm{V}_{u}$ are used for the unstable subspace modeling. The most unstable global mode

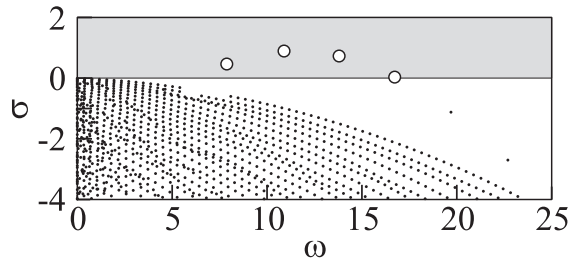

Figure 3 Least-stable part of the global spectrum. Unstable modes are shown by empty signs and stable modes are shown in black is displayed in Fig. $4 a$.

The stable modes are represented by the black dots. These modes are used to model the stable subspace. In this case, they will be cast into the $(n \times p)$ matrix $\mathrm{V}_{s}$ where $p$ is the dimension of the stable subspace. Note that the adjoint basis $W$ verifies the adjoint eigenvalue problem: $A^{*} W=Q W \bar{\Lambda}$. 


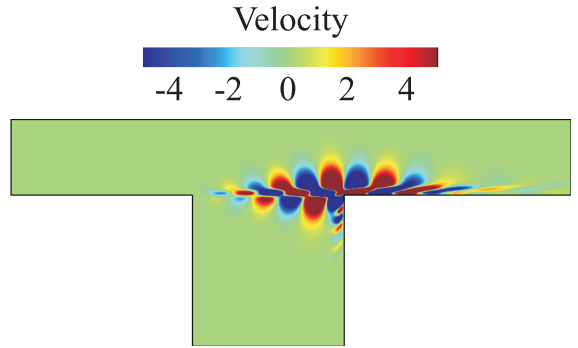

(a)

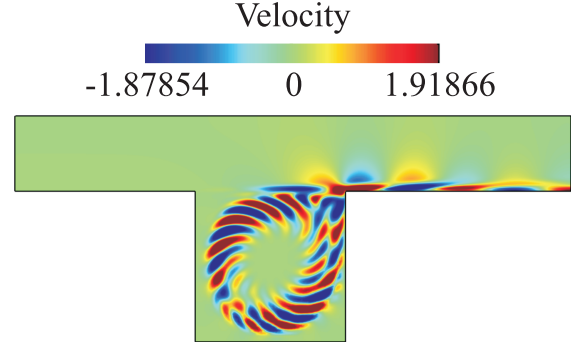

(b)

Figure 4 Unstable $(a)$ and stable (b) global modes (longitudinal velocity). (Refer Barbagallo et al., p. 506.)

\subsection{Proper Orthogonal Decomposition Modes}

Defined on the stable subspace, the POD modes are the most controllable structures, i. e., the states more easily triggered by the actuator. They correspond to the eigenvectors of the controllability gramian defined as:

$$
\mathrm{G}_{C}=\int_{0}^{\infty} e^{\mathrm{A} t} \mathrm{CC}^{*} e^{\mathrm{A}^{*} t} d t
$$

where the integrand is restricted to the stable subspace.

The POD modes are computed using a snapshot method [7]:

- 1000 equispaced snapshots from the impulse response of the direct problem (with the control function as initial condition) are cast into the $(n \times 1000)$ matrix:

$$
\mathbf{T}=\left[\begin{array}{lll}
\mathbf{X}(0 \Delta t) \sqrt{\delta_{0}} & \mathrm{X}(1 \Delta t) \sqrt{\delta_{1}} & \ldots
\end{array}\right]
$$

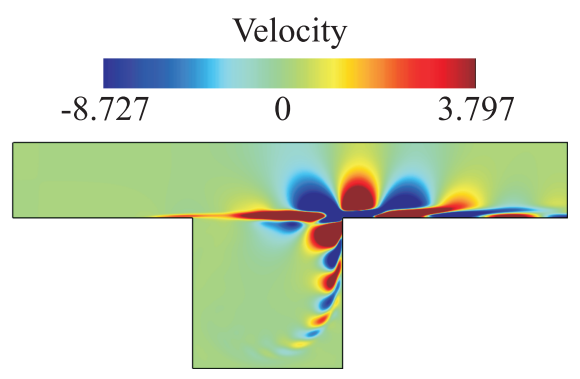

(a)

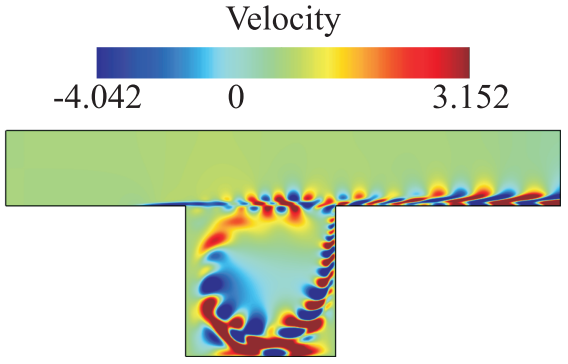

(b)

Figure 5 First $(a)$ and 28th $(b)$ POD modes (longitudinal velocity). (Refer Barbagallo et al., p.506.) 
- a singular value decomposition (SVD) of the matrix $T^{*} Q T$ is performed: $\mathrm{T}^{*} \mathrm{QT}=\mathrm{T}^{\prime} \Sigma \mathrm{T}^{*} ;$ and

- the POD modes are given by $\mathrm{V}=\mathrm{TT}^{\prime} \Sigma^{-1 / 2}$.

The first and 28th POD modes are displayed in Fig. 5 by contour of longitudinal velocity.

\subsection{Balanced Proper Orthogonal Decomposition Modes}

Since controllable (POD) modes can be hardly observable, they can be inappropriate in a compensated problem where an estimated flow field is made using the information arising from a sensor. For this reason, the modes which maximize both controllability and observability are considered. These modes, also defined on the stable subspace, are found as the eigenvectors of the matrix $G_{C} G_{O}$ where $\mathrm{G}_{O}$ is the observability gramian defined as:

$$
\mathrm{G}_{O}=\int_{0}^{\infty} e^{\mathrm{A}^{*} t} \mathrm{M}^{*} \mathrm{M} e^{\mathrm{A} t} d t
$$

Again, the integrand is restricted to the stable subspace.

The BPOD modes are computed using a snapshot method [5]:

- 1000 equispaced snapshots from the impulse response of the direct problem (with the control function as initial condition) are cast into the $(n \times 1000)$ matrix:

$$
\mathbf{T}=\left[\begin{array}{lll}
\mathbf{X}(0 \Delta t) \sqrt{\delta_{0}} & \mathrm{X}(1 \Delta t) \sqrt{\delta_{1}} & \ldots
\end{array}\right]
$$

- 1000 equispaced snapshots from the impulse response of the adjoint problem (with the measurement function as initial condition) are cast into the $(n \times 1000)$ matrix:

$$
\mathrm{U}=\left[\begin{array}{lll}
\mathrm{Y}(0 \Delta t) \sqrt{\delta_{0}} & \mathrm{Y}(1 \Delta t) \sqrt{\delta_{1}} & \ldots
\end{array}\right]
$$

- an SVD of the matrix $\mathrm{U}^{*} \mathrm{QT}$ is performed: $\mathrm{U}^{*} \mathrm{QT}=\mathrm{U}^{\prime} \Sigma \mathrm{T}^{\prime *}$;

- the direct BPOD modes are given by $\mathrm{V}=\mathrm{TT}^{\prime} \Sigma^{-1 / 2}$; and

- the adjoint BPOD modes are given by $\mathrm{W}=\mathrm{UU}^{\prime} \Sigma^{-1 / 2}$.

The first and 13th BPOD modes are displayed in Fig. 6 by contour of longitudinal velocity. 


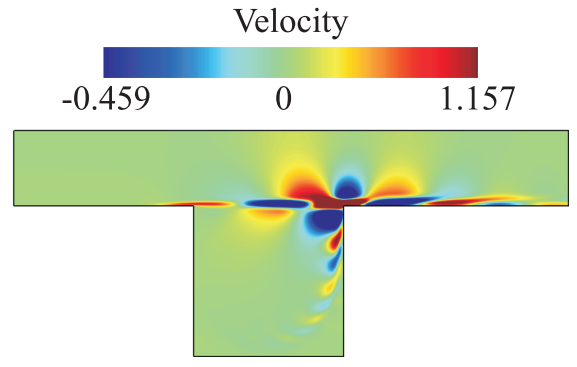

(a)

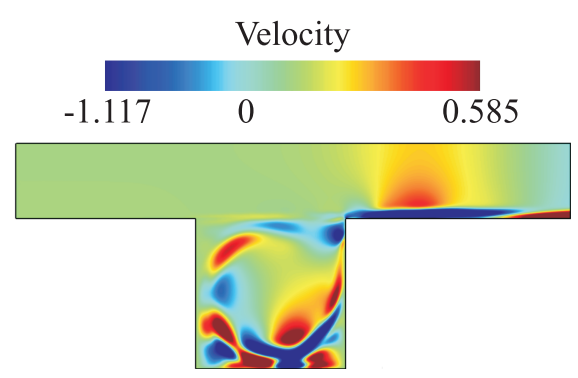

(b)

Figure 6 First $(a)$ and 13th (b) BPOD modes (longitudinal velocity). (Refer Barbagallo et al., p. 508.)

\section{INPUT-OUTPUT BEHAVIOR OF THE STABLE SUBSPACE}

In this section, the authors aim at evaluating how accurately the input-output behavior of the stable subspace is captured by each of the reduced models. This is done using systematic tools such as transfer functions and error norms. In the whole section, only the stable dynamics will be considered.

Consider a reduced model of the form:

$$
\begin{aligned}
\frac{d \hat{X}}{d t} & =\hat{A} \hat{X}+\hat{C}_{c} ; \\
m & =\hat{M} \hat{X} .
\end{aligned}
$$

The transfer function is defined as $\hat{\mathrm{H}}(\omega)=\hat{\mathrm{M}}(i \omega \hat{\mathrm{I}}-\hat{\mathrm{A}})^{-1} \hat{\mathrm{C}}$. It is computed for each reduced model and compared in Figs. $7 a-7 c$ with the transfer function of the full system. The latter is the Fourier transform of the measurement signal from an impulse response with the control function as initial condition. A quantitative comparison is obtained using the $\mathcal{H}_{\infty}$ norm defined as

$$
\|\mathrm{G}(\omega)\|_{\infty}=\sup _{\omega}|\mathrm{G}(\omega)| \text {. }
$$

In Fig. $7 d-7 f$, the relative $\mathcal{H}_{\infty}$-error $\|\hat{\mathrm{H}}-\mathrm{H}\|_{\infty} /\|\mathrm{H}\|_{\infty}$ is plotted vs. the number of stable modes accounted for in the ROM.

For reduced models based on global modes, the main peak is captured using reduced models containing $p=132,1201$, and 3000 . However, when more modes are added (see $p=4000$ ), the transfer function deteriorates. This is in agreement with the error norm which oscillates when $p<4000$ and eventually increases drastically. This behavior can be related to the nonnormality of the linearized Navier-Stokes operator. In this case, the highly damped modes become very 


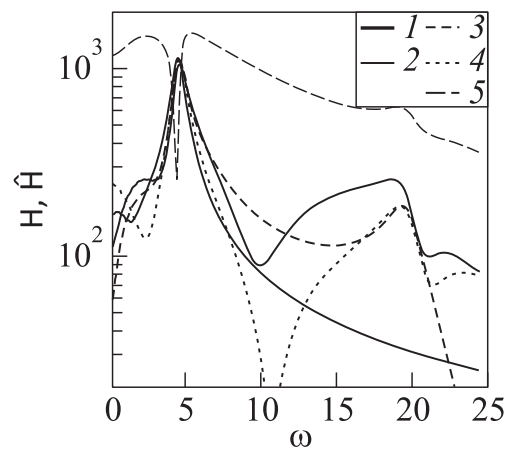

(a)

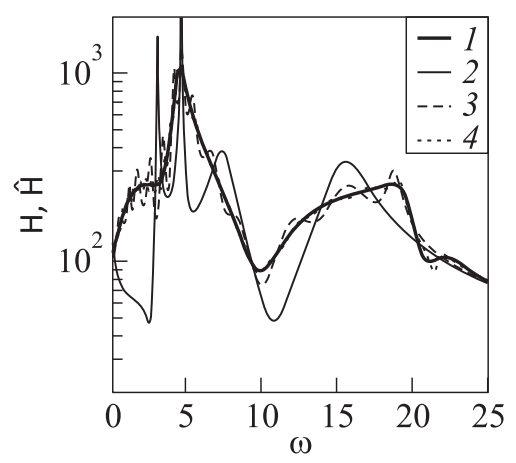

(b)

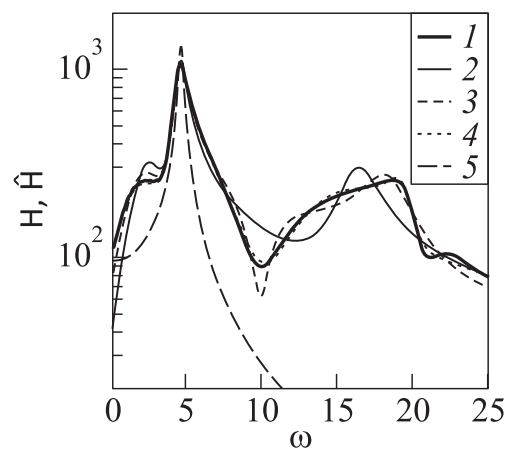

(c)

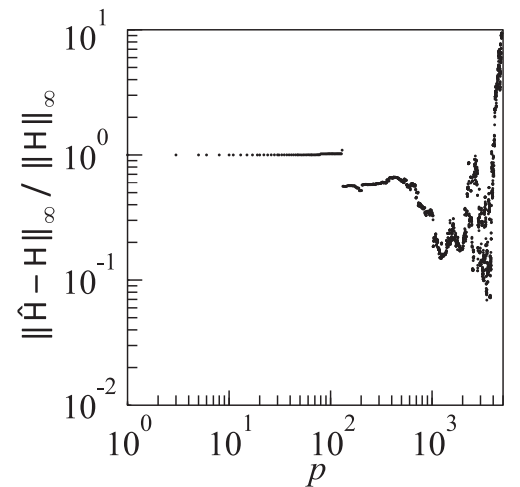

(d)

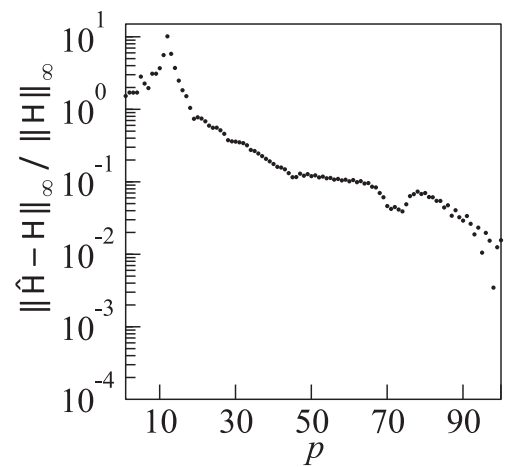

(e)

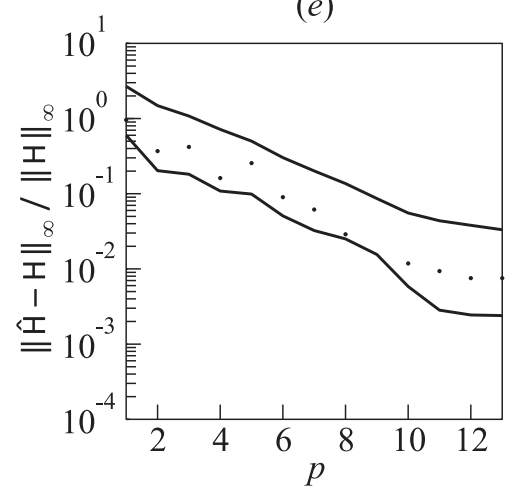

$(f)$

Figure 7 Comparison of transfer function of the ROM to the exact (1) transfer function for a projection basis of various order, using global modes $(a)(2-p=132$; $3-1201 ; 4-3000$; and $5-p=4000)$, POD modes $(b)(2-p=10 ; 3-28$; and $4-p=50)$, and BPOD modes $(c)(2-p=2 ; 3-6 ; 4-8$; and $5-$ $p=10)$. Relative $\mathcal{H}_{\infty}$-error norm as a function of the number $p$ of included modes, using global $(d)$, POD $(e)$, and BPOD modes $(f)$ 
sensitive to the action of the actuator when the ROM is constructed using a bi-orthogonal projection [8]. To overcome this feature, alternative projection techniques have been considered [9].

When POD modes are used in the reduced model, the transfer function is well approximated provided a sufficient number of modes is retained. This feature is observed again when BPOD modes are used; however, with a smaller number of modes, only 10 BPOD modes are necessary to obtain an error norm of $10^{-2}$, although nearly 100 POD modes are required to achieve a similar performance.

So, reduced models based on global modes (using the bi-orthogonal projection) do not capture the input-output relation whatever the number of modes considered. On the contrary, reduced models based on BPOD modes achieve this goal, so as POD modes with a much higher number of modes.

\section{LINEAR QUADRATIC GAUSSIAN CONTROL AND COMPARISON}

After evaluating how reduced models based on global, POD, and BPOD modes model the stable subspace, let come back to the full problem of the stabilization of a globally unstable flow.

An LQG control strategy where the controller and the estimator are designed separately according to the separation principle is considered. The controller is computed assuming that the variable $\hat{X}$ is known for all time. It links the control law $c$ and the reduced field according to a linear relation $c=\hat{\mathrm{K}} \hat{\mathrm{X}}$. When the entire state is not known and only a measure of the state is available (so-called partial state control), the estimator yields an approximation $\hat{Y}$ of the reduced field $\hat{X}$. In this case, the control law is computed using $c=\hat{K} \hat{Y}$ (see details in [10]). The reader should remember that the controller and estimator are based on the reduced model and not on the full system.

In Fig. 8, the energy of the compensated flow is represented vs. time for reduced models of varying order ( $p$ represents the number of modes modeling the stable subspace). Note that no results with reduced models based on stable global modes are shown since the compensated system was always found unstable.

For reduced models based on POD and BPOD modes, the system is unstable if only a few modes are considered. When more modes are added, the amplification rate of the energy decreases, ultimately leading to a stable system. This performance is related to an improved capture of the input-output behavior as the number of modes increases. In agreement with the previous section, BPOD modes are much more efficient in stabilizing the flow with a small number of modes.

As shown in [8], the stability of the compensated system using an arbitrary reduced model can be investigated replacing the linearized direct numerical simulation (plant) by a reduced model (thereafter called the reduced plant) which 


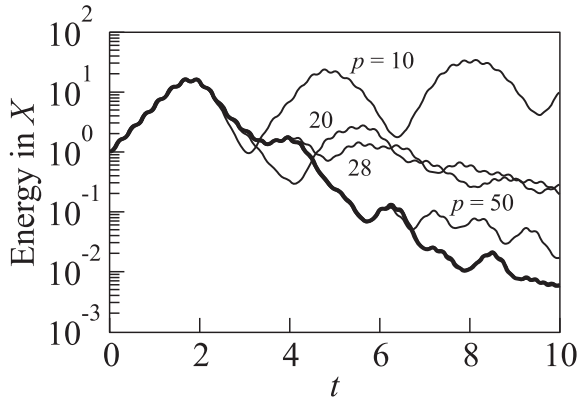

(a)

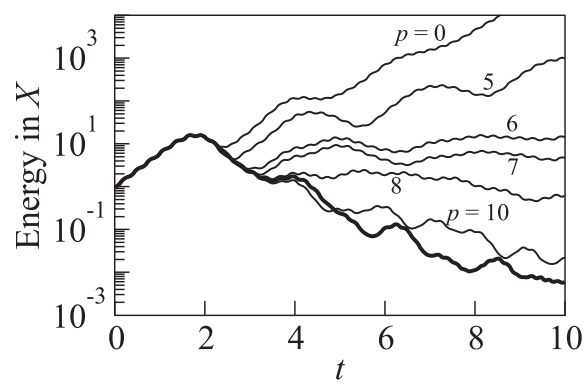

(b)

Figure 8 Performance of partial-state controller measured as the perturbation kinetic energy vs. time for different ROMs: $(a)$ the number of included POD modes (in addition to the unstable global modes) is denoted by $p$; and $(b)$ the number of included BPOD modes (in addition to the unstable global modes) is denoted by $p$

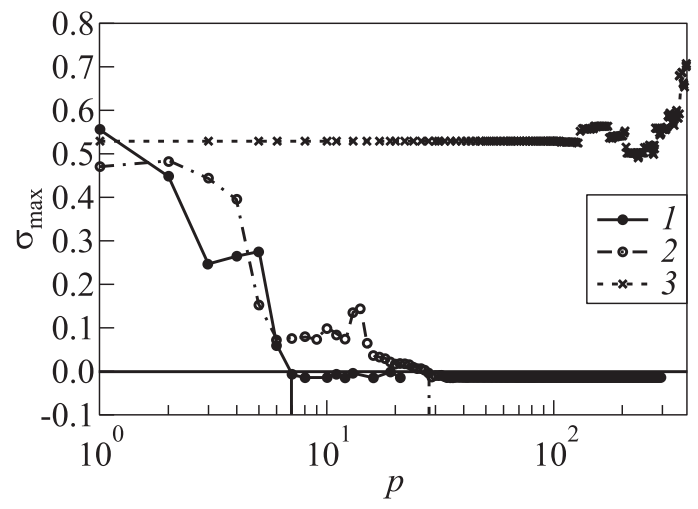

Figure $9 \sigma_{\max }$ vs. the number of modes modeling the stable subspace for reduced models based on BPOD (1), POD (2), and global (3) modes

captures accurately the input-output relation. In this case, a reduced model composed of 8 unstable modes and 13 BPOD modes is considered. Considering the eigenvalues of the coupled system: reduced plant + compensator based on an arbitrary reduced model, a positive real part of the least stable eigenvalue $\sigma_{\max }$ indicates an unstable compensated system. This quantity is displayed in Fig. 9 (black dots) vs. the number $p$ of modes modeling the stable subspace. While only 7 BPOD modes are required to stabilize the system, reduced models based on POD modes need at least 28 modes to achieve this goal (see the dashed vertical line). Whatever the number of global modes used, they cannot stabilize the system. This is in agreement with the energy curves in Fig. 8. 


\section{CONCLUDING REMARKS}

In this paper, a closed-loop control based on various reduced models have been considered to suppress a global instability. It is shown that in order to stabilize the flow, the reduced model needs not only to span the unstable subspace, but also to capture accurately the input-output behavior of the stable subspace. The latter condition is not verified by reduced models based on global modes (using the bi-orthogonal projection) whatever the number of modes included in the reduced model. This makes them unable to achieve a successful closed-loop control. On the contrary, provided a sufficient number of modes are considered for the modeling of the stable subspace, the input-output behavior is well approximated by reduced models based on BPOD or POD modes. In this case, the perturbations are suppressed. Moreover, the superiority of BPOD modes on POD modes, i. e., their ability to control with a fewer number of modes, is attributed to their improved observability.

\section{REFERENCES}

1. Åkervik, E., J. Hoepffner, U. Ehrenstein, and D. S. Henningson. 2007. Optimal growth, model reduction and control in a separated boundary-layer flow using global modes. J. Fluid Mech. 579:305-14.

2. Delville, J., L. Cordier, and J. P. Bonnet. 1998. Large-scale structure identification and control in turbulent shear flows. Flow control: Fundamentals and practice. Springer Verlag. 199-273.

3. Ilak, M., and C.W. Rowley. 2008. Modeling of transitional channel flow using balanced proper orthogonal decomposition. Phys. Fluids 20:034103.

4. Bagheri, S., L. Brandt, and D. S. Henningson. 2008. Input-output analysis, model reduction and control of the flat-plate boundary layer. J. Fluid Mech. 620:263-98.

5. Rowley, C. W. 2005. Model reduction for fluids using balanced proper orthogonal decomposition. Int. J. Bifurc. Chaos 15(3):997-1013.

6. Sipp, D., and A. Lebedev. 2007. Global stability of base and mean flows: a general approach and its applications to cylinder and open cavity flows. J. Fluid Mech. 593:333-58.

7. Sirovich, L. 1987. Turbulence and the dynamics of coherent structures. Quarter. Appl. Math. XLV(3):561-90.

8. Barbagallo, A., D. Sipp, and P. J. Schmid. 2009. Closed loop control of an open cavity flow using reduced-order models. J. Fluid Mech. 641:1-50.

9. Ehrenstein, U., P. Y. Passaggia, and F. Gallaire. 2010. Control of a separated boundary layer: Reduced-order modeling using global modes revisited. Theor. Comput. Fluid Dyn. 25(1-4):195-207.

10. Bewley, T.R., and S. Liu. 1998. Optimal and robust control and estimation of linear paths to transition. J. Fluid Mech. 365:305-49. 